\title{
Reflexões sobre a Capacitação Gerencial na Agricultura Familiar Brasileira
}

Reflections on management in the Brazilian Family Farming

\author{
Joelsio José Lazzarotto ${ }^{8}$ \\ João Caetano Fioravanço
}

\section{Resumo}

A agricultura familiar apresenta grande relevância para o desenvolvimento social e econômico do Brasil. No entanto, a ampla maioria dos produtores rurais familiares apresenta sérias deficiências gerenciais. Diante disso, baseando-se principalmente em análises de estudos técnico-científicos, buscou-se efetuar reflexões acerca da gestão nos estabelecimentos de agricultura familiar, dando-se ênfase a questões relacionadas com a situação gerencial e com o modelo e os mecanismos que possam ampliar a capacidade de gerenciamento nessas organizações. Dentre os principais resultados e conclusões, destaca-se que, para minimizar os problemas gerenciais dos agricultores familiares, a utilização da visão sistêmica e o envolvimento efetivo desses atores organizacionais são imprescindíveis em todas as etapas de planejamento e execução do modelo de capacitação a ser adotado.

Palavras-chave: Tecnologias de gestão. Enfoque sistêmico. Metodologia participativa.

\section{Abstract}

Family farming in Brazil has great relevance to the social and economic development. However, the vast majority of family farmers has serious managerial deficiencies. Thus, this work aimed to make reflections on managing family farms, emphasizing the managerial situation and the model and the mechanisms to improve management capacity in these

8 Joelsio José Lazzarotto: Médico veterinário, mestre em administração rural, doutor em economia aplicada e pesquisador da área de socioeconomia da Empresa Brasileira de Pesquisa Agropecuária - Embrapa. Áreas de interesse em pesquisa: tomada de decisão na agricultura, estudos de sistemas de produção agropecuária, avaliação de tecnologias e análises de resultados econômico-financeiros de empreendimentos agroindustriais. Vínculo institucional: Embrapa Uva e Vinho.Email: joelsio@cnpuv.embrapa.br.

João Caetano Fioravanço:Engenheiro Agrônomo, mestre em Fitotecnia, doutor em Economia, Sociologia e Política Agrícola (Agronegócios) e pesquisador da Empresa Brasileira de Pesquisa Agropecuária - Embrapa. Áreas de interesse em pesquisa: fruticultura, fisiologia e manejo de plantas, seleção de variedades e sistemas de produção sustentáveis. Vínculo institucional: Embrapa Uva e Vinho. E-mail: fioravanco@cnpuv.embrapa.br. 
organizations. As results and conclusions, we emphasize that, to minimize management problems of farmers, the systemic approach and the effective involvement of these organizational actors are essential in all phases of planning and implementing the training model to be adopted.

Keywords: Management technologies. Systemic approach. Participatory methodology.

\section{Introdução}

A agricultura familiar constitui um segmento de fundamental relevância para o desenvolvimento social e econômico do Brasil. Essa importância pode ser justificada por dois pontos principais: $84 \%$ das propriedades rurais do País são familiares; e os estabelecimentos familiares respondem, respectivamente, por $34 \%$ e $74 \%$ do valor bruto e do pessoal ocupado na produção agropecuária nacional (CENSO..., 2006).

Apesar dessa relevância, a agricultura familiar brasileira depara-se com grandes problemas, que constituem fortes entraves para a sua competitividade e sustentabilidade ao longo do tempo. Entre esses problemas, merecem atenção especial aqueles associados com aspectos de gestão, pois, de maneira geral, a ampla maioria dos pequenos e médios produtores tem sérias deficiências gerenciais, elevando, assim, a frequência de empreendimentos familiares mal remunerados.

Portanto, a questão gerencial é um fator crítico para o desenvolvimento da agricultura familiar nacional. Diante disso, é imprescindível que a extensão rural melhore suas estratégias de ação para transmitir aos produtores familiares importantes noções gerenciais, incluindo aspectos de planejamento, controle, comercialização e análise econômica da produção. Com essas noções, o produtor poderá tomar decisões mais rápidas e eficientes, tornando o seu negócio mais competitivo (BUAINAIN; BATALHA, 2007) e sustentável, independente do seu tamanho (REICHERT, 1998).

Partindo dessa contextualização inicial e levando-se em conta que na literatura nacional ainda são escassos os trabalhos técnico-científicos que envolvem, ao mesmo tempo, avaliações situacionais e discussões sobre ações de capacitação gerencial de agricultores familiares, foi elaborado este artigo. O objetivo principal consiste em efetuar reflexões sobre a gestão nos estabelecimentos de agricultura familiar, enfatizando-se, principalmente, questões relacionadas com a situação gerencial dos produtores familiares e com o modelo e os mecanismos que podem ser usados para ampliar a sua capacidade de gerenciamento.

\section{A situação gerencial na agricultura familiar brasileira}

Um empreendimento rural, familiar ou não, deve ser gerido eficientemente como forma de garantir sua inserção no mercado e, por consequência, sua sustentabilidade e competitividade (BATALHA et al., 2004; LOURENZANI, 2006). Essa afirmação é plenamente justificável pelo fato de que existe uma 
série de fatores que podem afetar significativamente o desempenho das propriedades rurais, como: o ciclo produtivo que, além de irreversível, é dependente de condições biológicas; as condições climáticas que condicionam a maior parte das explorações agropecuárias; o caráter perecível da maioria dos produtos agropecuários que interfere nos processos de comercialização, armazenamento e conservação; e os altos custos de saída e/ou entrada em um empreendimento agropecuário (SOUZA et al., 1995).

Apesar de muitos desses fatores não serem controláveis, outros podem ter algum controle mediante a utilização de tecnologias gerenciais adequadas. Essas tecnologias incluem novas formas de negociação e práticas de gestão do processo produtivo, possibilitando, ao produtor rural, melhorias no processo de tomada de decisão (UECKER et al., 2005; LOURENZANI, 2006).

Embora as questões gerenciais sejam imprescindíveis para o sucesso de qualquer empreendimento, é consenso que, enquanto os aspectos vinculados com a produção (insumos, operações agrícolas etc.) são considerados parte da rotina operacional da maioria dos estabelecimentos rurais familiares, a utilização rotineira de instrumentos de gestão (aspectos comerciais e contábeis, planilhas de resultados etc.) tende a ser exceção nessas organizações (REZENDE; ZYLBERSZTAJN, 1999; SANDRI, 2003; BATALHA et al., 2004; NEUKIRCHEN et al., 2005; LOURENZANI, 2006; MARION; SEGATI, 2006).

As dificuldades de gerenciamento enfrentadas pelos agricultores familiares tendem a perpetuar-se, pois expressiva parcela desses atores organizacionais, além de não receber auxílio gerencial adequado (BUAINAIN; BATALHA, 2007), apresentam grande descapitalização, não conseguindo, portanto, ter acesso e beneficiar-se de modernas tecnologias de informação. Nessa mesma linha, Batalha et al. (2004) assinalam que, no Brasil existe um esforço considerável no desenvolvimento e difusão de tecnologias de processo, de materiais e de produtos e serviços. Por outro lado, os esforços voltados para as tecnologias de gestão e informação direcionadas, sobretudo, para os pequenos produtores rurais são ainda muito escassos e, via de regra, inócuos.

Relacionado com os trabalhos técnico-científicos desenvolvidos no País e que envolvem aspectos de gestão de empreendimentos rurais, é pertinente salientar que, além de muito incipientes, a grande maioria tende a ser difundida apenas em forma de publicações, não estando vinculada a ações efetivas de capacitação gerencial de produtores e técnicos extensionistas. A partir de Mercês e Sant'Ana (2005), pode-se inferir que essa capacitação constitui outro grande entrave, especialmente associado aos programas de assistência técnica e extensão rural, que estão entre os principais meios que os produtores familiares utilizam para obter orientações de diversas naturezas.

De acordo com Batalha et al. (2004), os mecanismos de difusão tecnológica adotados no Brasil não têm sido suficientes para capacitar o agricultor familiar na implementação e utilização das técnicas de gestão disponíveis. Mesmo junto a produtores que possuem alto grau de tecnificação produtiva, é pobre o emprego de técnicas adequadas de 
gerenciamento. Para Segatti e Hespanhol (2008), o ritmo intenso da atualização tecnológica no campo requer a atualização de informações e a adoção de mecanismos de aprendizagem e de formação profissional dos agricultores. Essa exigência tem penalizado muitos pequenos produtores, sistematicamente desalojados do ambiente onde estão inseridos devido à necessidade de se produzir em grande quantidade, com elevado padrão de qualidade e a preços competitivos.

\section{Modelo de capacitação gerencial na agricultura familiar}

Ações de capacitação gerencial de produtores familiares devem incluir diversos aspectos, como planejamento estratégico e controle e análise de custos de produção. Porém, para que essas ações propiciem os resultados esperados, Zuin e Zuin (2007) enfatizam que é importante que o capacitador traduza conhecimentos advindos da academia à "língua" falada dos aprendizes, ou seja, o diálogo como essência da relação educacional deve problematizar o conhecimento dentro da sua realidade concreta, a fim de melhor compreendê-la, explicá-la e transformá-la.

O modelo de capacitação, com uma abordagem participativa, territorial e multidisciplinar, contemplando conhecimentos técnicos e gerenciais, deve estar adaptado às condições locais, considerando as peculiaridades do público-alvo e da região (LOURENZANI, 2006). A necessidade de adoção de um modelo dessa natureza deve-se ao fato de que, na maioria das regiões agropecuárias do Brasil, existem e coexistem diferentes e complexos sistemas de produção. Essa diferenciação e complexidade devem-se, principalmente, às diferenças na disponibilidade, qualidade e utilização dos fatores de produção (terra, capital, trabalho e tecnologia) e às formas como os produtores aceitam as inovações tecnológicas, concordam e/ou executam tarefas e se relacionam com o mercado e as demais variáveis que compõem o ambiente organizacional externo (ZORDAN; GONÇALVES, 1995; LAZZAROTTO et al., 2004). Em outras palavras, o referido modelo deve estar focado em um processo de aprendizagem e de construção coletiva de conhecimento, que parta de respostas relacionadas com a seguinte questão: que conhecimentos gerenciais os agricultores familiares inseridos em determinada realidade necessitam se apropriar para alcançar o desenvolvimento sustentável? Portanto, o ponto de partida e chegada para a construção do conhecimento em gestão organizacional é o homem em sua atividade real, onde a formação administrativa não é um ritual abstrato, acadêmico e elitista, mas se inscreve na própria vida e prática dos agricultores, tornando possível a integração do processo educativo ao processo produtivo desenvolvido por eles (LIMA et al., 1995).

Diante dessas considerações, é possível afirmar que as ações de capacitação precisam estar sustentadas na visão sistêmica, que, em termos práticos, possibilita realizar levantamentos da situação socioeconômica dos agricultores, identificando e caracterizando os principais sistemas de produção, as práticas técnicas, sociais e econômicas e os problemas enfrentados. Permite, ainda, caracterizar o desenvolvimento rural presente e avaliar tendências para agricultura regional como forma de projetar a 
evolução da realidade agrária e propor políticas, programas, projetos e ações de desenvolvimento prioritários, que sejam mais adaptados aos contextos social, econômico e agroecológico em que vivem os agricultores (INCRA/FAO, 1999; DUFUMIER, 1996).

A utilização do enfoque sistêmico, além de não ignorar os objetivos socioeconômicos dos agricultores, é relevante para compreender problemas de relações, estruturas e interdependência das propriedades rurais com seu ambiente externo. Essa compreensão, de acordo com Dufumier (1996), constitui fator-chave para a proposição de intervenções adequadas a cada realidade rural, contrapondo o discurso dos pacotes tecnológicos, em que o agente da extensão rural levava ao agricultor uma proposta pronta. Esse fato exige que o extensionista compreenda a racionalidade dos agricultores e o porque de suas atitudes, visando a definir a forma como podem ser introduzidas mudanças que não provoquem impactos negativos ou desestruturem a organização interna da unidade produtiva (FAVERO; SARRIERA, 2009).

\section{Mecanismos operacionais para a capacitação gerencial}

Baseando-se nos fundamentos teóricos acerca do modelo de capacitação gerencial, para implementar ações efetivas direcionadas ao aprimoramento da gestão na agricultura familiar, propõe-se a adoção de mecanismos operacionais que contemplem cinco etapas chaves: caracterização geral das unidades de produção, definição de grupos de agricultores, identificação das demandas gerenciais prioritárias, desenvolvimento e validação conjunta de instrumentos metodológicos e implementação de ações de capacitação. Em todas essas etapas, o emprego da visão sistêmica e a ampla participação de representantes de extensionistas e de produtores são fundamentais para assegurar o adequado cumprimento das ações previstas.

A etapa de caracterização geral das unidades de produção consiste em obter um diagnóstico geral, que envolve cinco aspectos principais dos estabelecimentos agropecuários presentes em determinada realidade rural (LIMA et al., 1995). O primeiro refere-se à localização e ao tipo de inserção nos meios físico e socioeconômico. O segundo aspecto trata do meio agroecológico, que envolve basicamente a caracterização do meio natural. Quanto ao terceiro, ele contempla as características estruturais dessas organizações: grupo familiar e meios produtivos. O quarto aspecto diz respeito ao funcionamento dos sistemas produtivos, envolvendo análises dos usos dos recursos produtivos, bem como das práticas e técnicas que os agricultores adotam. Finalmente, o quinto aspecto consiste em estudar a trajetória das propriedades rurais, o que permite compreender o estágio em que se encontram, procurando identificar os fatores que, ao longo do tempo, influíram ou condicionaram o processo decisório dos produtores rurais.

Com base nos resultados obtidos com a caracterização das unidades produtivas, avança-se para a etapa de definição de grupos de agricultores. Nessa etapa são estabelecidos grupos menores de produtores que, em função de explorarem sistemas de produção agropecuária um tanto 
similares, tendem a apresentar características socioeconômicas e problemas gerenciais, também, similares. O estabelecimento desses grupos é reforçado, ainda, pelo fato de que além de existir grande limitação de agentes atuantes na extensão rural brasileira, a organização de agricultores impulsiona-os a aprenderem, de forma permanente, mediante trocas de experiências com outras pessoas. Com essa estratégia, os produtores podem fazer comparações entre as suas unidades de produção, percebendo as diferenças e semelhanças e relacionando as diferentes variáveis que interferem nos resultados físicos e econômicos do processo produtivo. Com isso, têm melhores subsídios para a tomada de decisões.

Após definir todos os grupos de produtores, pode-se avançar para a etapa de identificação das demandas gerenciais prioritárias. Essa identificação trata de avaliar, para cada grupo, os conhecimentos e as habilidades gerenciais que precisam ser aprimorados por meio de ações efetivas de capacitação.

Levando-se em conta as demandas, pode-se iniciar a etapa de desenvolvimento e validação conjunta de instrumentos metodológicos (e.g., cadernos de contabilidade simplificados) a serem utilizados na realização das ações em questão. Nesse sentido, a partir de discussões permanentes envolvendo, sobretudo, extensionistas e representantes de cada grupo de produtores familiares, esses instrumentos devem estar bem ajustados e adequados (facilidades de compreensão, utilização e aplicação) à realidade desses produtores. Os cuidados no desenvolvimento e na validação dos citados instrumentos são sustentados por Altieri (2002), pois este enfatiza que a geração de tecnologias adequadas às necessidades da agricultura familiar deve surgir de estudos integrados das condições ambientais e socioeconômicas, que influenciam os sistemas de produção e controlam suas respostas às alternativas tecnológicas.

A etapa de implementação de ações de capacitação representa o momento em que, para cada grupo de agricultores e com as demandas identificadas, serão desenvolvidas as ações específicas de capacitação. Nessa etapa, ao envolver aspectos de gerenciamento organizacional, primeiramente, devem ser estimuladas discussões gerais entre produtores e extensionistas, com o objetivo de resgatar a forma e os conhecimentos tradicionalmente empregados pelos agricultores na gestão das suas unidades produtivas, identificando, de forma conjunta, os principais pontos fortes e as deficiências mais acentuadas vinculadas ao processo gerencial. Após essas discussões, podem ser utilizados os instrumentos metodológicos específicos visando a aprofundar questões que contribuam para aprimorar a gestão organizacional.

É importante assinalar que, paralelamente à execução das cinco etapas descritas, deve-se efetuar permanente avaliação dos mecanismos operacionais e dos resultados obtidos. Essa avaliação é fundamental para permitir, quando necessária, a efetivação de correções de rumos, de maneira a não comprometer o processo de capacitação gerencial dos produtores. Além disso, para que esse processo propicie os resultados esperados, ele deve fazer parte de um plano que contemple ações continuadas, ou seja, ao invés de estar restrita a um curso ou uma palestra, a capacitação em discussão deve pautar-se na programação e no 
desenvolvimento de várias atividades. Com isso, busca-se fazer com que o produtor, de fato, introduza nas suas rotinas diárias e cultura gerencial os princípios e mecanismos administrativos abordados nas ações de capacitação, que podem durar, por exemplo, duas safras agrícolas. Ações continuadas são essenciais para que o agricultor melhor se familiarize com diversos aspectos, como planejamento e replanejamento de atividades e avaliações de metas e resultados.

Finalmente, cabe enfatizar que, na execução das cinco etapas citadas, podem ser empregadas várias técnicas metodológicas, entre as quais se destacam os painéis de discussão e os roteiros. Enquanto os painéis objetivam uma discussão crítica entre os participantes sobre assuntos de relevância, os roteiros são constituídos por vários tópicos e podem ser muito úteis para orientar as discussões com os produtores, evitando-se, assim, que questões relevantes deixem de ser abordadas.

\section{Considerações finais}

As discussões efetuadas ao longo do trabalho, além de contextualizar a situação da gestão na agricultura familiar brasileira, procuraram fornecer subsídios auxiliares aos serviços de assessoramento junto aos produtores familiares. Isso porque, como na prática a opção por implantar determinado sistema produtivo depende, fundamentalmente, das preferências e escolhas efetuadas pelo agricultor e seu grupo familiar, os serviços em questão precisam aprimorar as suas estratégias de atuação. Para tanto, a utilização da visão sistêmica e o envolvimento efetivo de produtores e extensionistas em todas as etapas de planejamento e execução das ações voltadas para a capacitação gerencial são imprescindíveis, especialmente para assegurar que sejam observadas e atendidas as reais necessidades dos principais beneficiários, os agricultores familiares.

$\mathrm{Na}$ condução das referidas ações, a formação de uma equipe multidisciplinar de assessoramento, também, é estratégica por duas razões. A primeira reside no fato de contribuir com melhorias importantes na comunicação e transferência de conhecimentos e tecnologias existentes, relacionados com diferentes aspectos técnicos e gerenciais que afetam os resultados da produção agropecuária. A segunda razão é decorrente do fato dessa equipe facilitar a elaboração de diversos instrumentos metodológicos específicos, que serão utilizados nas várias etapas do processo de capacitação gerencial.

De maneira sintética, baseando-se em Gibbons et al. (1994), é possível assinalar que, na capacitação gerencial de agricultores familiares, o modelo a ser empregado precisa ajustar-se ao modo emergente de produção do conhecimento, que apresenta algumas características fundamentais: 1) na organização, ao invés de centrar-se no contexto acadêmico e com visão disciplinar, deve desenvolver-se no contexto da aplicação a partir de uma visão multidisciplinar; 2) em termos de equipe, precisa deslocar-se de um grupo pequeno e homogêneo para um grupo grande e heterogêneo, caracterizando a estruturação de redes para atingir vários objetivos e interesses; 3) pelo lado da responsabilidade social, esta deve permear todo 
o processo de produção e aplicação do conhecimento; 4) sobre a questão da reflexividade, é necessário passar de uma visão predominantemente técnico-científica para uma visão em que há valorização de todo conhecimento útil à solução de determinado problema; e 5), finalmente, em relação ao controle de qualidade, ao invés de ser realizado por pares, deve envolver novos critérios (impactos esperados) e novos atores (clientes e beneficiários).

\section{Referências}

ALTIERI, M. Agroecologia: bases científicas para uma agricultura sustentável. Guaíba: Agropecuária, 2002. 592p.

BATALHA, M.; BUAINAIN, A.M.; SOUZA FILHO, H.M. de. Tecnologia de gestão e agricultura Familiar. In: CONGRESSO DA SOCIEDADE BRASILEIRA DE ECONOMIA E SOCIOLOGIA, 42., 2004, Cuiabá. Anais..., Cuiabá: SOBER, 2004. (1 CD-Rom).

BUAINAIN, M.A.; BATALHA, M.O. Cadeia produtiva de fruta. Brasília: IICA/MAPA/SPA, 2007. 102 p. (Agronegócios, v.7).

CENSO Agropecuário - 2006. Disponível em: <http://www.sidra.ibge.gov.br/bda>. Acesso em: 02 de agosto de 2011.

DUFUMIER, M. Les projets de développement agricole: manuel d'expertise. Paris/Wageningen: Karthala/CTA, 1996. 354p.

FAVERO, E.; SARRIERA, J.C. Extensão rural e intervenção: velhas questões e novos desafios para os profissionais. Cadernos de Psicologia Social do Trabalho, Porto Alegre, v.12, n.1, p.1-16. 2009.

GIBBONS, M.; LIMOGES, C.; NOWOTNY, H.; SCHWARTZMAN, S.; SCOTT, P.; TROW, M. The new production of knowledge: the dynamics of science and research in contemporary societies. London: Sage. 1994.

INCRA/FAO. Guia metodológico: diagnóstico de sistemas agrários. Brasília: INCRA/FAO, 1999. 58p. (Projeto de Cooperação Técnica).

LAZZAROTTO, J.J.; MELLO, H.C.; ROESSING, A.C. Grupos de agricultores para a tomada de decisões organizacionais: uma proposta metodológica. In: CONGRESSO DA SOCIEDADE BRASILEIRA DE ECONOMIA E SOCIOLOGIA, 42., 2004, Cuiabá. Anais..., Cuiabá: SOBER, 2004. (1 CDRom).

LIMA, A.P. de.; BASSO, N.; NEUMANN, P.S. et al. Administração da unidade de produção familiar: modalidades de trabalho com agricultores. ljuí: UNIJUÍ, 1995. 175p.

LOURENZANI, W.L. Capacitação gerencial de agricultores familiares: uma proposta metodológica de extensão rural. Organizações Rurais \& Agroindustriais, Lavras, v.8, n.3, p.313-322. 2006. 
MARION, J.C; SEGATTI, S. Sistema de gestão de custos nas pequenas propriedades leiteiras. Custos e @gronegócio on line, v.2, n.2. Jul./Dez. 2006. Disponível em: <www.custoseagronegocioonline.com.br>. Acesso em: 02 de agosto de 2011.

MERCÊS, D.L. das; SANT'ANA, A.L. Análise da extensão rural no Cinturão verde de llha Solteira (SP): as perspectivas dos produtores e técnicos. In: CONGRESSO DA SOCIEDADE BRASILEIRA DE ECONOMIA E SOCIOLOGIA, 43., 2005, Ribeirão Preto. Anais..., Ribeirão Preto: SOBER, 2005. (1 CD-Rom).

NEUKIRCHEN, L.C.; ZANCHET, A.; PAULA, G. de. Tecnologia de gestão e rentabilidade na pequena propriedade rural - estudo de caso. In: CONGRESSO DA SOCIEDADE BRASILEIRA DE ECONOMIA E SOCIOLOGIA, 43., 2005, Ribeirão Preto. Anais..., Ribeirão Preto: SOBER, 2005. (1 CD-Rom).

REICHERT, L.J. A administração rural em propriedades familiares. Teoria e Evidência Econômica, Passo Fundo, v.5, n.10, p.67-86. Mai. 1998.

REZENDE, C.L.; ZYLBERSZTAJN, D. Uma análise da complexidade do gerenciamento rural. In: SEMINÁRIOS EM ADMINISTRAÇÃO (SEMEAD), 4., 1999, São Paulo. Anais..., São Paulo: Pós-graduação em Administração da FEA-USP. 1999. Disponível em: $<$ http://www.ead.fea.usp.br/semead/4semead/artigos/Adm_geral/Rezende_e _Zylbersztajn.html>. Acesso em: 10 de agosto de 2011.

SANDRI, E. de A.M. Modelo de gestão por atividades para empresas de fruticultura de clima temperado. 2003. 148f. Dissertação (Mestrado em Engenharia de Produção) - Universidade Federal de Santa Catarina, Florianópolis, 2003

SEGATTI, S.; HESPANHOL, A.N. Alternativas para a geração de renda em pequenas propriedades rurais. In: ENCONTRO NACIONAL DE GRUPOS DE PESQUISA - ENGRUP, 4., 2008, São Paulo. Anais..., São Paulo: Agrária, 2008. p. 615-631.

SOUZA, R. de; GUIMARÃES, J.M.P; VIEIRA, G.; MORAIS, V.A.; ANDRADE, J.G. de. A administração da fazenda. 5.ed. São Paulo: Globo, 1995. $211 \mathrm{p}$.

UECKER, G.L.; UECKER, A.D.; BRAUN, M.B.S. A gestão dos pequenos empreendimentos rurais num ambiente competitivo global e de grandes estratégias. In: CONGRESSO DA SOCIEDADE BRASILEIRA DE ECONOMIA E SOCIOLOGIA, 43., 2005, Ribeirão Preto. Anais..., Ribeirão Preto: SOBER, 2005. (1 CD-Rom).

ZORDAN, M.S.; GONÇALVES, W.M. A importância da tipificação de empresas rurais. Caderno de administração rural, Lavras, v.7, n.2, p.137146. 1995.

ZUIN, P.B.; ZUIN, L.F.S. Proposta de um modelo de desenvolvimento de produtos para propriedades familiares fundamentado na metodologia de 
Revista Tecnologia e Sociedade - 1ª Edição, 2012.

ISSN (versão online): 1984-3526

Paulo Freire para extensão rural. Revista Tecnologia e Sociedade, Curitiba, n.5. p.49-60. Jul. 2007. 